\title{
离子液体为溶剂制备 $\mathrm{CMC} / \mathrm{MWCNTs}$ 复合材料 及其电化学性能
}

赵廷凯 ${ }^{(12 *}$, 刘乐浩 ${ }^{(1)}$, 李光明 ${ }^{(1)}$, 杜丽 ${ }^{(1)}$, 赵星 ${ }^{(1)}$, 颜进 ${ }^{(1)}$, 程有亮 ${ }^{(1)}$, 党阿䂞 ${ }^{(1)}$, 李铁虎 ${ }^{(1)}$

(1) 西北工业大学材料科学与工程学院, 西安 710072 ;

(2) Department of Materials Science and Engineering, Northwestern University, Evanston 60208, USA

* 联系人, E-mail: ztk-xjtu@163.com, ztk@nwpu.edu.cn

2011-12-30 收稿, 2012-01-19 接受

教育部博士学科点专项科研基金(20096102120016)、国家自然科学基金(51172184)、中国航天科技创新基金(CASC200906)和西北工业大学 基础研究基金(JC201030)资助

摘要 以羒甲基纤维素钠 (CMC) 和多壁碳纳米管( MWCNTs) 作为原料, 采用离子液体溶解羒 甲基纤维素钠制备 CMC/MWCNTs 复合材料。通过使用场发射扫描电子显微镜(FE-SEM)、高 分辨透射电子显微镜(HR-TEM)、X 射线衍射仪(XRD)和电化学工作站对复合材料进行了结 构表征和电化学性能分析. 结果表明, 以离子液体作为溶剂能够有效溶解 CMC 并使其较为 均匀地包覆在 MWCNTs 的表面, CMC 包覆层的厚度约 $5.4 \mathrm{~nm}$; 用离子液体制备的

关键词

多壁碳纳米管 羧甲基纤维素钠 离子液体 电化学性能 CMC/MWCNTs 复合材料对 $\mathrm{H}_{2} \mathrm{O}_{2}$ 具有良好的电催化效果, 在循环伏安曲线图中出现了明显 的氧化峰; MWCNTs 的含量和超声时间是影响复合材料分散性和电化学性能的关键参数, MWCNTs 的含量 $3 \mathrm{mg}$, 超声 $2 \mathrm{~h}$ 时, 制得的 CMC/MWCNTs 复合材料具有最优的电化学性能.

碳纳米管(carbon nanotubes, CNTs)自 1991 年由 Iijima $^{[1]}$ 以标志性的文章报道以来，由于其显著的物 化性能, 在电子器件、催化剂和生物医学等领域具有 潜在的应用前景, 吸引了科学家广泛的研究. 近年来, 以生物学应用为目的的碳纳米管/生物分子基复合材 料探索，即通过整合生物活性分子(酶、蛋白质、DNA 等)与碳纳米管的性能, 逐渐成为一个新的研究热 点 ${ }^{[2,3]}$. 而纤维素(cellulose)是一种天然的、可再生的高 分子材料, 它生长和存在于绿色植物中, 是一种取之 不尽、用之不竭的资源. 纤维素具有来源丰富、廉价 易得、生物相容性好等特点, 而碳纳米管具有独特的 物理和化学性能, 这使得纤维素/多壁碳纳米管复合材 料在生物材料、电化学等领域有很好的潜在应用 ${ }^{[4,5]}$.

离子液体 (ionic liquids, ILs)作为一类新兴物质, 与固体物质相比较, 它是液态的; 与传统的液态物质
相比较, 它是离子的; 与经典融熔盐相比较, 熔点较 低，通常不高于 $100^{\circ} \mathrm{C}$, 并具有热稳定性好、不燃烧、 不挥发、不氧化、不爆炸、低毒性等优点, 被视为环 境友好的绿色溶剂 ${ }^{[6-8]}$. 最近, Swatloski 等人 ${ }^{[9]}$ 报道 了离子液体溴化 1-丁基-3-甲基咪唑([BMIM]Br)能够 通过其阴离子与羟基的作用破坏纤维素分子间的氢 键, 从而溶解不经任何处理的纤维素, 引起了科学界 的关注. 因此, 离子夜体溶解的纤维素直接包覆到碳 纳米管表面, 制备一种有价格竞争力和生物相容性 的纳米复合材料将会有很好的应用价值.

纤维素/多壁碳纳米管复合材料作为一种新型复 合材料, 具有独特的结构和优良的物理化学性能. 它 结合了纤维素良好的生物相容性, 以及碳纳米管高 比表面、高强度等各自的优异性能, 是一种性能优异 的新型功能材料和结构材料, 在生物传感器和电化 Bull, 2012, 57: 1620-1625, doi: 10.1007/s11434-012-5009-2 
学领域将具有广阔的应用前景 ${ }^{[10 ~ 14]}$. 离子液体能够 通过其阴离子与羟基的作用破坏纤维素分子间的氢 键, 溶解不经任何处理的纤维素, 从而不会破坏纤维 素/碳纳米管复合材料的性质, 而且对于降低成本、减 少流程方面具有积极意义. 羧甲基纤维素钠 $(\mathrm{CMC})$ 是纤维素的一种钠盐, 目前羧甲基纤维素钠/多壁碳 纳米管 $(\mathrm{CMC} / \mathrm{MWCNTs})$ 生物复合材料的研究尚未见 有报道.

本文利用离子液体溶解羧甲基纤维素钠, 并将 其功能化到多壁碳纳米管表面, 制备了一种具有生 物相容性的羧甲基纤维素钠/多壁碳纳米管生物复合 材料, 重点讨论了其修饰电极对 $\mathrm{H}_{2} \mathrm{O}_{2}$ 的电催化作用, 分析了溶剂、碳纳米管含量、超声时间对 $\mathrm{CMC} /$ MWCNTs 复合材料的电化学性能的影响.

\section{1 实验与方法}

\section{1 实验原料}

多壁碳纳米管, 纯度 $>95 \%$, 平均直径 $11 \mathrm{~nm}$ (北 京天奈科技有限公司); 羧甲基纤维素钠(天津市东丽 区天大化学试剂厂); 浓硫酸、浓硝酸、吡啶、溴代 正丁烷、乙腈/乙酸乙酯均为分析纯, 购自国药集团 陕西化学试剂有限公司.

\section{2 实验方法}

(i ) 羧酸化碳纳米管. 将原始多壁碳纳米管 (p-MWCNTs) $0.5 \mathrm{~g}$ 置于雉形瓶中, 加人 $200 \mathrm{~mL}$ 浓硫 酸和浓硝酸(体积比 $3: 1$ ) 混合酸中, 在 $60^{\circ} \mathrm{C}$ 超声浴中 反应 $5 \mathrm{~h}$ 成均匀黑色溶液, 高速离心洗涤溶液至中性, 产物在 $100^{\circ} \mathrm{C}$ 真空烘箱中干燥 $8 \mathrm{~h}$, 得到羧基化碳纳 米管(f-MWCNTs).

(ii) 离子液体(ionic liquids, ILs)的制备. 将 $40.0 \mathrm{~g}$ 吡啶加人到 $250 \mathrm{~mL}$ 单颈圆底烧瓶中, 用翻口 橡皮塞密封, 然后反复抽充 3 次氮气, 在室温磁摚拌 条件下, 用微量注射器向烧瓶中分批加人 $75.4 \mathrm{~g}$ 澳代 正丁烷，然后水浴加热至 $50^{\circ} \mathrm{C}$ 继续磁摚拌 $12 \mathrm{~h}$. 将产 物加人到 $200 \mathrm{~mL}$ 乙酸乙酯中, 进行振荡、洗涤和分 液操作, 然后重复此操作 5 次. 再将产物放人 $200 \mathrm{~mL}$ 乙腈中静置、重结晶, 最后放人电热恒温鼓风干燥箱 进行真空干燥，并密封保存.

(iii) CMC/MWCNTs 的制备. 将 $1.0 \mathrm{mg}$ MWCNTs 和 $20 \mathrm{~g}$ 离子液体加人到 $100 \mathrm{~mL}$ 单颈圆底烧瓶中, 在 $80^{\circ} \mathrm{C}$ 下超声 $1 \mathrm{~h}$. 然后加人 $20 \mathrm{~g}$ 含 $\mathrm{CMC}$ 质量分数为 $5 \%$ 的离子液体溶液，继续超声 $1 \mathrm{~h}$ 后加人 $20 \mathrm{~mL}$ 去离 子水. 再将反应液用 $0.22 \mu \mathrm{m}$ 混纤滤膜进行抽滤，将 得到的黑色产物重新分散到去离子水中后再次进行 抽滤，反复此操作 5 次. 最后将制得的 $\mathrm{CMC} /$ MWCNTs 复合材料分散到 $5 \mathrm{~mL}$ 去离子水中待用.

将 $\mathrm{CMC} / \mathrm{MWCNTs}$ 复合材料配制在水溶液中, 超声分散 $10 \mathrm{~min}$, 形成浓度为 $10 \mathrm{mg} / \mathrm{mL}$ 稳定的悬浊 液. 以微量进样器取 $2 \mu \mathrm{L} \mathrm{MWCNTs/CS}$ 混合液置于 经抛光处理后的玻碳电极表面上, 经过红外灯烘干后, 即得到纤维素钠/多壁碳纳米管/玻碳电极(MWCNTs/ CS/GCE).

\section{3 表征方法}

采用 PANalystal 公司 X' Pert PRO 型 XRD 衍射 分析仪(Co K $\alpha$, 荷兰) 对 CMC/MWCNTs 复合材料进 行结构分析; SUPRA ${ }^{\mathrm{TM}} 55$ 型场发射扫描电子显微镜 (FE-SEM, 加速电压 $5 \mathrm{kV}$, 德国)分析复合材料的微 观特征; 高分辨透射电子显微镜 (HR-TEM, JEM3010 , 加速电压为 $300 \mathrm{kV}$, 日本)分析碳纳米管微观 结构特征; CHI650C 电化学工作站(上海辰华仪器有 限公司)分析电化学性能, 采用三电极系统(参比电极 为饱和甘录电极, 辅助电极为铂丝电极, 工作电极为 玻碳电极).

\section{2 结果与讨论}

\subsection{SEM 分析}

图 1(a)中可见，纯化的 MWCNTs 的表面没有任 何覆盖物, 比较平滑; 而从图 1(b)中可以观察到, CMC/MWCNTs 复合物仍呈管状, 但管壁包覆了一层 聚合物显得比较粗糙, 形成的复合物有一定程度的 缠结, 这说明超声处理对 CMC/MWCNTs 复合材料 的制备具有促进作用.

\subsection{HR-TEM 分析}

图 2(a)中可以观察到纯化的 MWCNTs 大都管壁 光滑致密、石墨片层清晰，没有明显的无定型碳和催 化剂粒子存在；在图 2(b)中可以观察到厚度比较均匀 的聚合物包覆在 MWCNTs 的表面，以及结构比较紧 密的 MWCNTs 管壁和相对比较松散的聚合物层, 从 图中可以测出 $\mathrm{CMC}$ 包覆层的平均厚度约为 $5.4 \mathrm{~nm}$. 

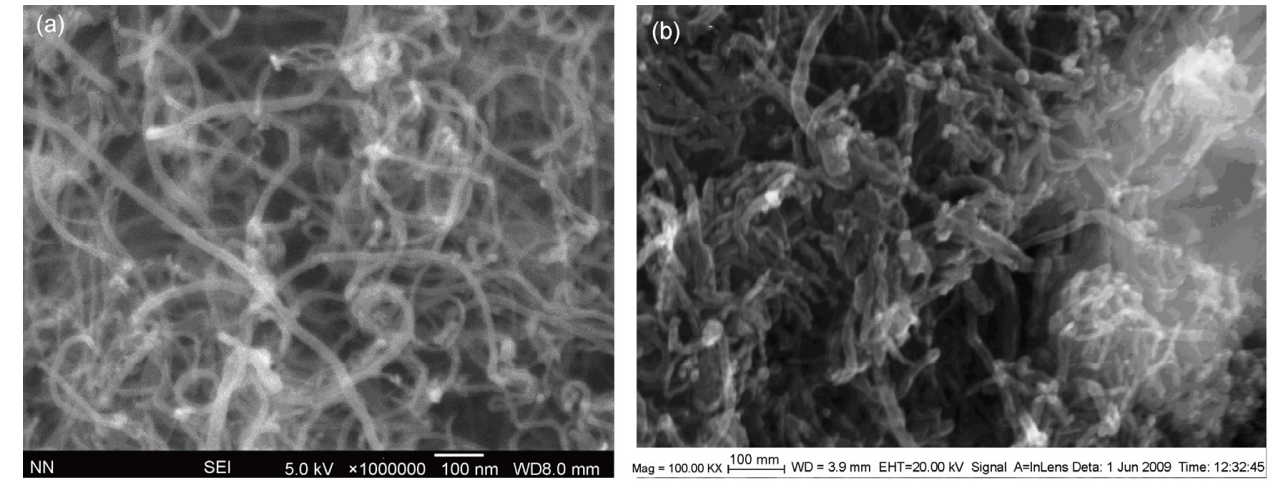

图 1 纯化的 MWCNTs(a)和 CMC/MWCNTs 复合材料(b)的 SEM 照片 MWCNTs 含量 $3 \mathrm{mg}$, 超声处理 $2 \mathrm{~h}$

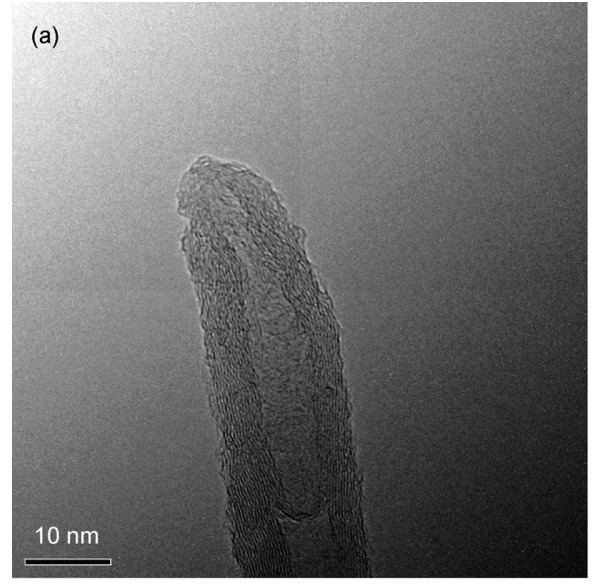

(b)

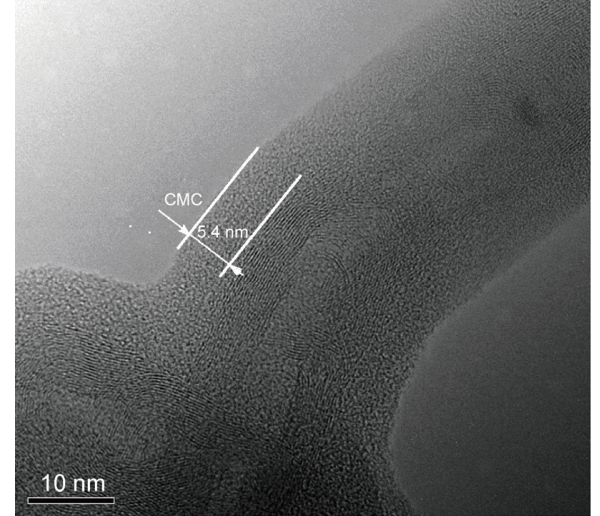

图 2 纯化的 MWCNTs(a)和 CMC/MWCNTs(b) 的 HR-TEM 照片

如果 CMC 能够均匀地包覆在 MWCNTs 的表面, 将 会形成一种核-壳的结构, 即 MWCNTs 为核、CMC 为壳. MWCNTs 层与 CMC 层的致密、有序程度不同, 使能够借助 HR-TEM 来清楚观察 CMC/MWCNTs 复 合材料.

\section{$2.3 \mathrm{XRD}$ 分析}

CMC/MWCNTs 复合材料在 $11^{\circ}$ 和 $30^{\circ}$ 处有 CMC 特征衍射峰, 并且含有一些官能团小峰; 而 $26.5^{\circ}$ 处 有 MWCNTs 特征衍射峰, 如图 3 所示. XRD 图谱说 明该复合材料中含有 CMC 和 MWCNTs.

\section{4 分散性对比}

图 4 为 $\mathrm{CMC} / \mathrm{MWCNTs}$ 溶液分散性对比照片, 超 声时间分别为 $2 \mathrm{~h}(\mathrm{~A}), 1 \mathrm{~h}(\mathrm{~B})$ 和 $0.5 \mathrm{~h}(\mathrm{C})$. 可以看出, 超声处理时间的增加能够促进 CMC 和 MWCNTs 的 复合反应. 超声 $2 \mathrm{~h}$ 的 CMC/MWCNTs 在水中的溶解 分散性明显比超声 0.5 和 $1 \mathrm{~h}$ 复合材料的分散性好, 在室温下静置 $72 \mathrm{~h}$ 后仍保持均匀分散状态; 而超声 时间为 0.5 和 $1 \mathrm{~h}$ 的 CMC/MWCNTs 复合材料均出现 了大块的团聚和沉淀，其中超声时间为 $0.5 \mathrm{~h}$ 的沉淀

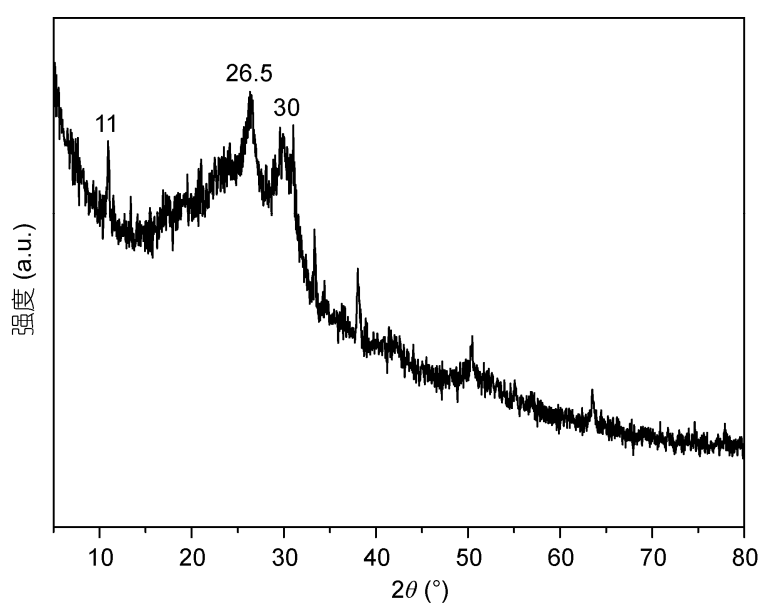

图 $3 \mathrm{CMC} / \mathrm{MWCNTs}$ 复合材料 XRD 图谱 MWCNTs 含量 $3 \mathrm{mg}$, 超声处理 $2 \mathrm{~h}$ 
(a)

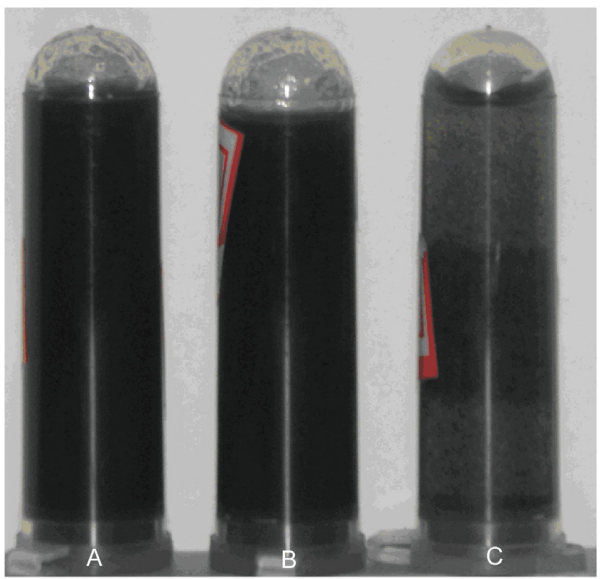

(b)

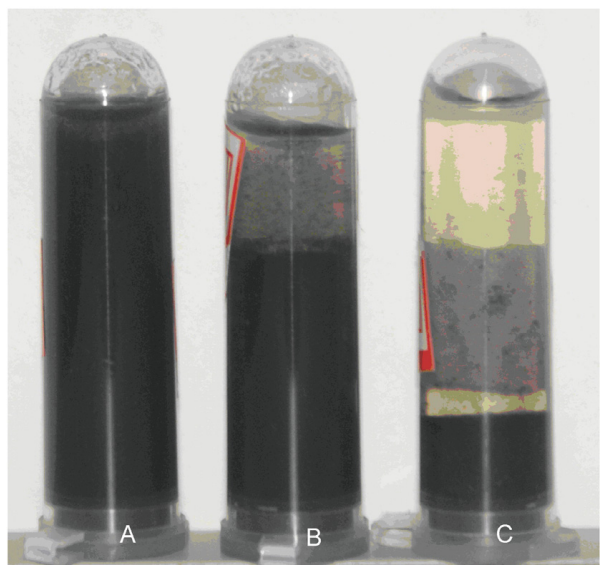

图 4 CMC/MWCNTs 溶液分散性照片

(a) 静置 $40 \mathrm{~min}$; (b) 静置 $72 \mathrm{~h}$

更为明显. 包覆在 MWCNTs 上的 CMC 含有大量的 羟基等极性基团，容易和水发生亲水作用，使得 MWCNTs 能够稳定的分散在水中. 超声处理能够促 进 CMC 的溶解，使更多的 CMC 包裹在 MWCNTs 的 表面，从而有效地提高了 $\mathrm{CMC} / \mathrm{MWCNTs}$ 的分散性.

\section{5 电化学性能}

$\mathrm{H}_{2} \mathrm{O}_{2}$ 是很多工业过程常用的原料或中间产物, 同时也是生物体内许多氧化酶反应的副产物，因而 其含量测定在食品、药物、临床、工业和环境分析中 具有重要的实际意义. 电化学分析法因具有较高的 灵敏度、良好的线性范围、快速的响应而被广泛使用. 本文采用制备的 CMC/MWCNTs 复合材料来修饰玻碳 电极, 进而讨论该 CMC/MWCNTs/GCE 复合材料在

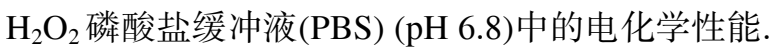

图 5 是不同修饰电极在 $10 \mathrm{mmol} / \mathrm{L}$ 的 $\mathrm{H}_{2} \mathrm{O}_{2} / \mathrm{PBS}$ 中的循环伏安曲线图 $(C V \mathrm{~s})$. 从图中可以观察到, 裸

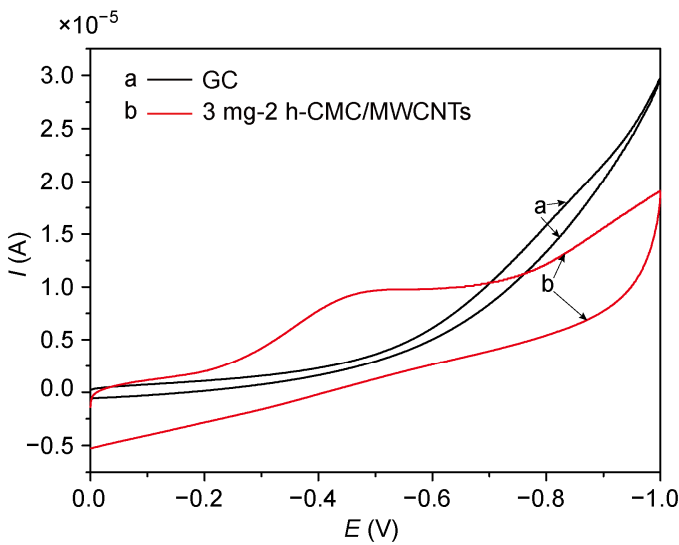

图 5 不同修饰电极在 $10 \mathrm{mmol} / \mathrm{L}$ 的 $\mathrm{H}_{2} \mathrm{O}_{2} / \mathrm{PBS}$ 中的循环 伏安曲线图

扫描速率 $0.04 \mathrm{~V} / \mathrm{s}$

的玻碳电极 (GCE) 在 $\mathrm{H}_{2} \mathrm{O}_{2} / \mathrm{PBS}$ 中没有出现任何氧化 还原峰, 而 CMC/MWCNTs/GCE 在-0.47 V 附近出现 了氧化电流, 形成氧化峰. 这是因为 $\mathrm{CMC} / \mathrm{MWCNTs} /$ GCE 上含有大量的 MWCNTs 和 CMC, 而且碳纳米 管具有优良的电化学特性, 能促进溶液中电活性物 质的电子交换; 另外 $\mathrm{CMC}$ 上的一些活性基团(如差甲 基等)对某些物质具有良好的吸附、鳌合、交换等作 用; 同时复合材料表面的多孔结构，使得修饰电极的 表面结构发生了根本的变化, 有利于物质的富集和 小分子的扩散. 因此, CMC/MWCNTs/GCE 对 $\mathrm{H}_{2} \mathrm{O}_{2}$ 的电催化性能得到了提高.

图 6 为在不同溶剂中制得的 CMC/MWCNTs 复 合材料修饰电极在 $\mathrm{H}_{2} \mathrm{O}_{2} / \mathrm{PBS}$ 中的循环伏安曲线图. 从图中可以看出, 以去离子水为溶剂制得的 $\mathrm{CMC} /$

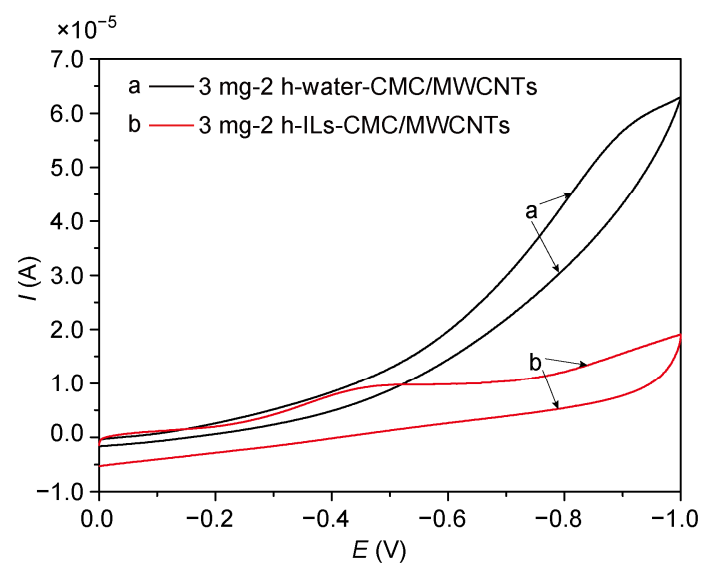

图 6 不同溶剂制得 CMC/MWCNTs 复合材料修饰的电极 在 $10 \mathrm{mmol} / \mathrm{L}$ 的 $\mathrm{H}_{2} \mathrm{O}_{2} / \mathrm{PBS}$ 中的 $C V \mathrm{~s}$ 图

扫描速率 $0.04 \mathrm{~V} / \mathrm{s}$ 
MWCNTs 复合材料修饰电极在 $\mathrm{H}_{2} \mathrm{O}_{2} / \mathrm{PBS}$ 中没有出 现任何氧化峰, 而以离子液体为溶剂制得的 $\mathrm{CMC} /$ MWCNTs 复合材料修饰电极则出现明显的氧化峰. 这是因为离子液体具有高的极性, 并能够充分地溶 解羧甲基纤维钠, 使离子液体溶解的纤维素能够更 好地包覆到碳纳米管的表面上, 从而得到功能化良 好的 CMC/MWCNTs 复合材料.

图 7 为不同碳纳米管含量的 CMC/MWCNTs 复 合材料修饰电极在 $\mathrm{H}_{2} \mathrm{O}_{2} / \mathrm{PBS}$ 中的 $C V \mathrm{~s}$ 图. 从图中可 知, 只有 MWCNTs 含量为 $3 \mathrm{mg}$ 时, 修饰电极循环伏 安曲线出现明显的氧化峰; 当 MWCNTs 含量分别 为 1,5 和 $2,4 \mathrm{mg}$ 时, 所对应的 CMC/MWCNTs/GCE 循环伏安曲线趋势类似. 这可归因于 MWCNTs 和 $\mathrm{CMC}$ 在复合材料中起着同等且至关重要的作用, 当 MWCNTs 含量太少时, CMC 会严实地包裹在 MWCNTs 上, 并充分填充于 MWCNTs 孔隙之中, 这 种致密的包覆大幅度地降低了复合材料的电催化性 能. 而当 MWCNTs 含量太多时, 包裏在 MWCNTs 上 的 CMC 就会很少, 碳纳米管的分散性降低, 团聚显 著增加, 使得 $\mathrm{CMC}$ 的电化学活性不能充分地表现出 来, 从而影响复合材料的电催化性能. 因此, 本文选 取 MWCNTs 重量为 $3 \mathrm{mg}$ 作为最佳碳纳米管添加量.

从图 8 可知, 在相同的实验条件下, 只有超声时 间为 $2 \mathrm{~h}$ 时, 修饰电极出现明显的氧化峰; 当超声时 间分别为 $1,3 \mathrm{~h}$ 和 $1.5,2.5 \mathrm{~h}$ 时, 所对应的 $\mathrm{CMC} /$ MWCNTs/GCE 循环伏安曲线趋势类似, 而且都出现 了氧化峰. 可能的原因是超声时间太长时, $\mathrm{CMC}$ 会被 充分溶解并严实地填充在 MWCNTs 孔隙之中和包裹

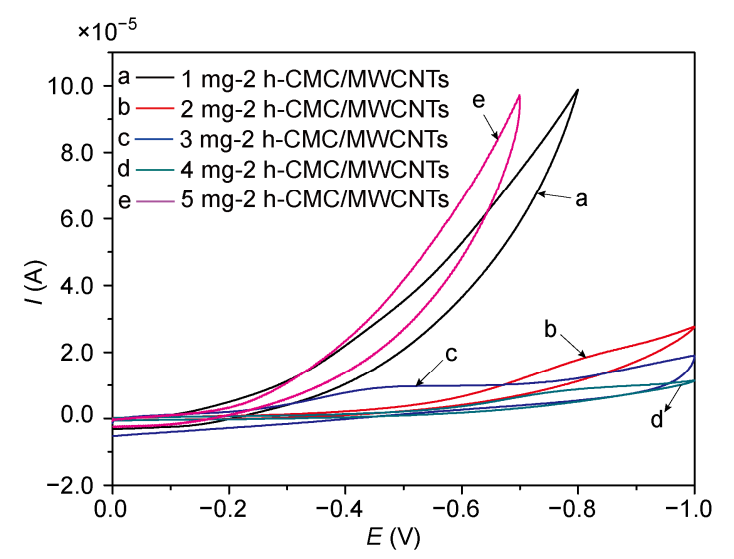

图 7 不同碳纳米管含量的 CMC/MWCNTs 复合材料修饰的 电极在 $10 \mathrm{mmol} / \mathrm{L}$ 的 $\mathrm{H}_{2} \mathrm{O}_{2} / \mathrm{PBS}$ 中的 $C V s$ 图 扫描速率 $0.04 \mathrm{~V} / \mathrm{s}$

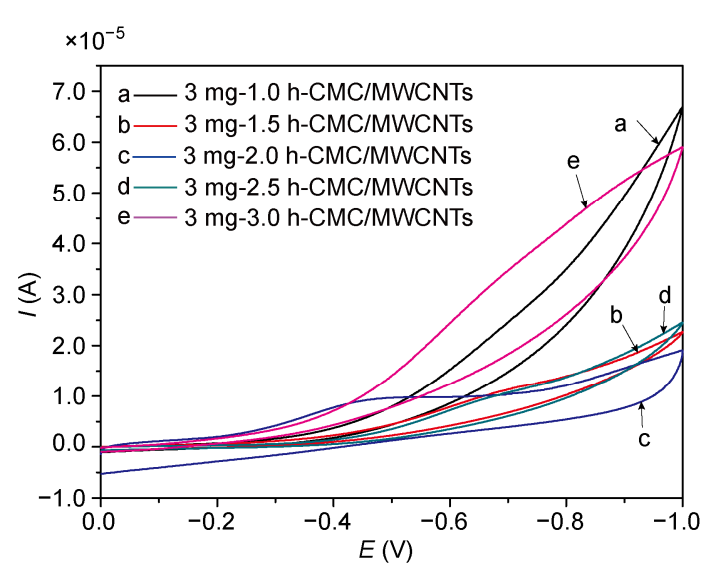

图 8 不同超声时间下制得的 CMC/MWCNTs 复合材料修饰 的电极在 $10 \mathrm{mmol} / \mathrm{L}$ 的 $\mathrm{H}_{2} \mathrm{O}_{2} / \mathrm{PBS}$ 中的 $C V \mathrm{~s}$ 图 扫描速率 $0.04 \mathrm{~V} / \mathrm{s}$

在 MWCNTs 上, 使得复合材料非常致密, 不利于 $\mathrm{H}_{2} \mathrm{O}_{2}$ 的扩散; 且长时间的超声处理还会破坏 $\mathrm{CMC}$ 上 的活性基团(环氧基、羧甲基等), 从而影响了复合材 料的电催化性能; 相反地如果超声时间太短, CMC 不能充分地溶解, 包裹在 MWCNTs 上的 CMC 就会 很少, 则 $\mathrm{CMC}$ 的电化学活性也就不能充分地表现出 来, 从而影响复合材料的电化学性能. 超声时间的长 短会改变 $\mathrm{CMC}$ 的溶解性和分散性, 从而改变 $\mathrm{CMC}$ 对 MWCNTs 的包裹、填充效果, 进而影响 CMC/ MWCNTs/GCE 的电催化性能. 因此, CMC/MWCNTs 复合材料分散性和溶解性的好坏直接影响复合电极 的电化学性能.

$\mathrm{H}_{2} \mathrm{O}_{2}$ 的氧化峰电流随着扫描速率的增大逐渐提 高(图 9), 表明 CMC/MWCNTs 复合材料修饰的 GCE

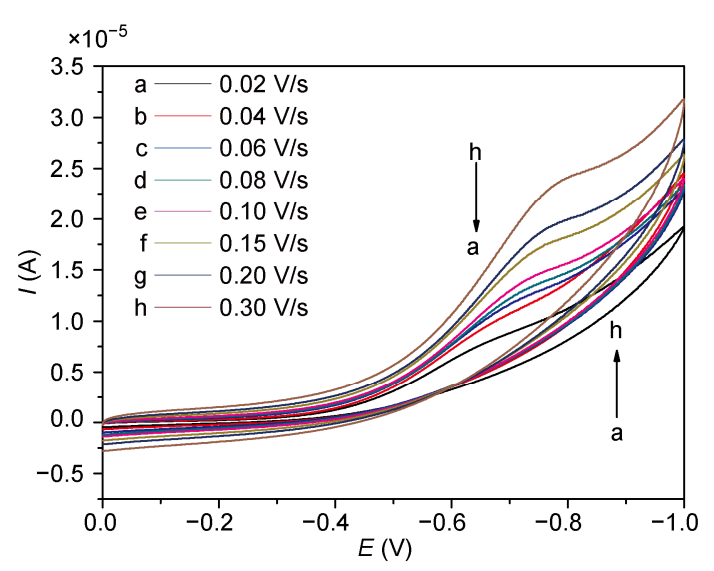

图 9 不同扫描速率下的 CMC/MWCNTs 复合材料的修饰电 极在 $10 \mathrm{mmol} / \mathrm{L}$ 的 $\mathrm{H}_{2} \mathrm{O}_{2} / \mathrm{PBS}$ 中的 $C V \mathrm{~s}$ 图 CNTs 含量 $3 \mathrm{mg}$, 超声时间 $2.5 \mathrm{~h}$ 
对 $\mathrm{H}_{2} \mathrm{O}_{2}$ 具有较好的电催化作用, 且在扫描速率为 $0.04 \mathrm{~V} / \mathrm{s}$ 时, 峰电流增加明显.

\section{3 结论}

（1）通过密封的水浴加热装置制备离子液体, 用 溶于离子液体的羧甲基纤维素钠功能化多壁碳纳米 管, 制备出水溶性的 CMC/MWCNTs 复合材料. CMC 能够比较均匀地包覆在 MWCNTs 的表面, 当 MWCNTs 含量为 $3 \mathrm{mg}$, 超声处理 $2 \mathrm{~h}$ 时, 制备的复合 材料的包覆效果最好, $\mathrm{CMC}$ 包覆层的厚度约为 $5.4 \mathrm{~nm}$.

(2) 超声时间能够改变 CMC 对 MWCNTs 的
包裏、填充效果，进而影响 CMC/MWCNTs 复合材料 的分散性和电化学性能. 离子液体对制备电化学性 能良好的 $\mathrm{CMC} / \mathrm{MWCNTs}$ 复合材料起着至关重要的 作用.

(3) CMC 包覆在 MWCNTs 的表面, 改变了其表 面结构, 使 CMC/MWCNTs/GCE 对 $\mathrm{H}_{2} \mathrm{O}_{2}$ 表现出良好 的电催化特性, 而且 MWCNTs 的含量和超声处理的 时间很大地影响了 CMC/MWCNTs/GCE 对 $\mathrm{H}_{2} \mathrm{O}_{2}$ 的 电催化效果. 在 MWCNTs 的含量为 $3 \mathrm{mg}$, 超声时间 为 $2 \mathrm{~h}$ 时, 所制得的 CMC/MWCNTs 复合材料具有最 优的电化学性能.

\section{参考文献}

1 Iijima S. Helical microtubules of graphitic carbon. Nature, 1991, 354: 56-58

2 Esawi A M K, Farag M M. Carbon nanotube reinforced composites: Potential and current challenges. Mater Des, 2007, 28: 2394-2401

3 Kocharova N, Leiro J, Kankare J, et al. Aqueous dispersion, surface thiolation, and direct self-assembly of carbon nanotubes on gold. Langmuir, 2007, 23: 3363-3371

4 Edgar K J, Buchanan C M, Debenham J S, et al. Advances in cellulose ester performance and application. Prog Polym Sci, 2001, 26: $1605-1688$

5 Yun S, Kim J. Multi-walled carbon nanotube-cellulose paper for a chemical vapor sensor. Sens Actuator B-Chem, 2010, 150: 308-313

6 Fukushima T, Kosaka A, Ishimura Y, et al. Molecular ordering of organic molten salts triggered by sing-wadded carbon nanotubes. Science, 2003, 300: 2072-2074

7 Welton T. Room-temperature ionic liquids solvents for synthesis and catalysis. Chem Rev, 1999, 99: 2071-2082

8 Huddleston J G, Willauer H W, Swatloski R P, et al. Room temperature ionic liquids as novel media for "Clean" liquid-liquid extraction. Chem Commun, 1998, 1765-1766

9 Swatloski R P, Spear S K, Holbrey J D, et al. Dissolution of cellose with ionic liquids. J Am Chem Soc, 2002, 124: 4974-4975

10 田蓉, 李少卿, 万丽, 等. 离子液体在碳纳米管功能化及复合材料中的应用研究进展. 化工进展, 2008, 27: 1569-1573

11 伏传龙, 孟令杰, 路庆华. 离子液体为溶剂制备纤维素/单壁碳纳米管复合物. 材料工程, 2008, (8): 26-30

12 Adagouda M N, Varma R S. Mictowave assisted synthesis of crosslinked poly(vinyl alcohol) nanocomposites comprising singlewalled carbon nanotubes, multi-walled carbon nanotubes, and buckminsterfullerene. Macromol Rapid Commun, 2007, 28: 842-847

13 Ukushima T, Aida T. Ionic liquids for soft functional material with carbon nanotubes. Chem Eur J, 2007, 13: 5048-5058

14 Marcilla R, Curri M L, Cozzoli P D, et al. Nano-obiects on a round trip from water to organics in a polymeric ionic liquid vehicle. Small, 2006, 2: 507-512 